\title{
Endovascular Angioplasty for Extracranial Vertebral Artery Occlusion Without Visualization of the Stump of the Artery Ostium
}

\author{
-Technical Note-
}

\author{
Kouhei NII, ${ }^{1}$ Gorou ABE, ${ }^{1}$ Minoru IKO, ${ }^{1}$ Yasuyuki NOMOTO, ${ }^{1}$ \\ Iwae YU, ${ }^{1}$ Kimiya SAKAMOTO, ${ }^{2}$ and Kiyoshi KAZEKAWA ${ }^{2}$
}

${ }^{1}$ Department of Neurosurgery, Fukuoka Kieikai Hospital, Fukuoka, Fukuoka;

${ }^{2}$ Department of Neurosurgery, Fukuoka University Chikushi Hospital, Chikushino, Fukuoka

\begin{abstract}
An 87-year-old man presented with extracranial vertebral artery (VA) occlusion and progressive vertebrobasilar ischemia despite maximal medical management. Cerebral angiography showed left proximal VA occlusion, termination of the right VA at the ipsilateral posterior inferior cerebellar artery, and hypoplastic bilateral posterior communicating arteries. Although the stump of the left VA ostium was not visualized, the distal patent artery was reconstituted via muscular branches from the left subclavian artery (SCA). Endovascular angioplasty with a stent for left VA occlusion was performed. The nonvisualized VA ostium was extrapolated from the computed tomography angiography findings of the distal patent VA and the partial calcification of the SCA. The occluded VA was penetrated by the guide wire and revascularized by balloon angioplasty with the stent using the support of a snare wire inserted via the left brachial artery for stabilization of the guide catheter. This treatment resulted in resolution of the severe neurological findings.
\end{abstract}

Key words: extracranial vertebral artery occlusion, endovascular angioplasty, progressive cerebral infarction, snare wire, computed tomography angiography

\section{Introduction}

Proximal extracranial vertebral artery (VA) disease is a common cause of vertebrobasilar ischemia and an embolic source in the posterior circulation. ${ }^{1,22}$ After the initial occurrence of untreated vertebrobasilar stroke or transient ischemic attack, the risk of stroke within the following 5 years is $25-35 \%{ }^{13)}$ Percutaneous endovascular treatment of extracranial VA disease is not as technically challenging as surgical procedures, and as such, endovascular angioplasty is a valuable alternative..$^{2,7,9,16,20,21)}$ Common endovascular angioplasty is performed after a wire device is navigated across the lesion. However, if the origin of the VA is not visualized, manipulation of the wire may be difficult. Here, we report a technical case of endovascular angioplasty based on computed tomography (CT) angiography for extracranial VA occlusion without visualization of the stump of the artery ostium.

Received February 8, 2012; Accepted June 7, 2012

\section{Illustrative Case}

An 87-year-old man presented with dysarthria and right hemiparesis. His past medical history was significant for hypertension and congestive heart failure. Diffusion-weighted magnetic resonance (MR) imaging showed small high intensity areas in the left pons and cerebellar hemisphere. Although MR angiography showed the basilar artery, the left VA was occluded at the ostium, and the right VA terminated at the ipsilateral posterior inferior cerebellar artery (PICA) (Fig. 1A). The patient was given dual anti-platelet therapy with aspirin $(100 \mathrm{mg} /$ day $)$ and cilostazol (200 mg/day), together with edaravone, ozagrel, and statin for acute cerebral infarction. Despite maximal medical management, alterations in consciousness and right hemiparesis progressively worsened. Follow-up diffusionweighted MR imaging showed progressive infarction including the pons, midbrain, and bilateral cerebellar hemispheres (Fig. 1B). CT angiography revealed an extracranial left VA occlusion, but the 

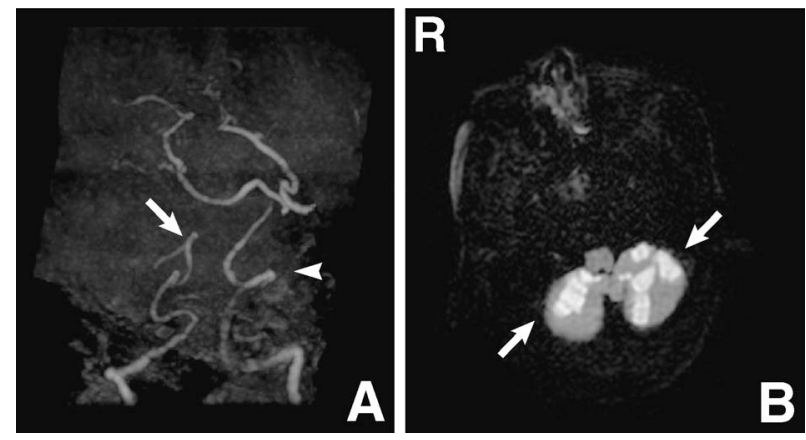

Fig. 1 A: Magnetic resonance (MR) angiogram showing the indistinct basilar artery (arrowhead) and the right vertebral artery that terminates at the ipsilateral posterior inferior cerebellar artery (arrow). B: Followup diffusion-weighted MR image showing progressive infarction including the brainstem and bilateral cerebellar hemispheres (arrows).

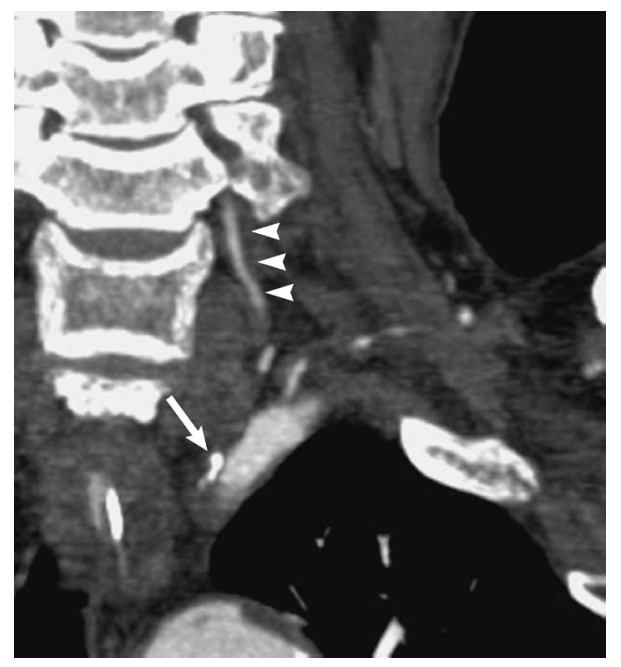

Fig. 2 Cervical computed tomography angiogram (maximum intensity projection image, anteroposterior view) showing proximal vertebral artery occlusion, distal patent vertebral artery (arrowheads), and partial calcification of the subclavian artery (arrow).

stump of the artery ostium was not visualized (Fig. 2). We performed cerebral angiography to confirm these findings and to develop a treatment strategy.

Cerebral angiography showed occlusion of the extracranial left VA without visualization of the stump of the artery ostium, which was reconstituted via branches of the thyrocervical artery (Fig. 3). The right hypoplastic VA terminated at the ipsilateral PICA, and the bilateral posterior communicating arteries were not visualized (Fig. 4A). Thus, the blood supply to the vertebrobasilar system was via the muscular and thyrocervical collateral vessels (Fig.
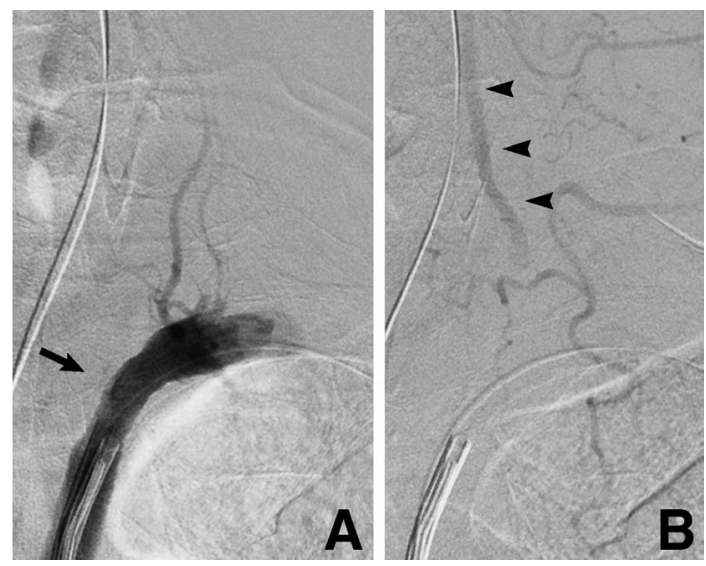

Fig. 3 Preoperative left subclavian artery angiograms (A: early phase, B: late phase) showing left vertebral artery occlusion (arrow). The stump of arterial ostium is not visualized. The distal patent artery was reconstituted via the muscular branches and thyrocervical branches (arrowheads).
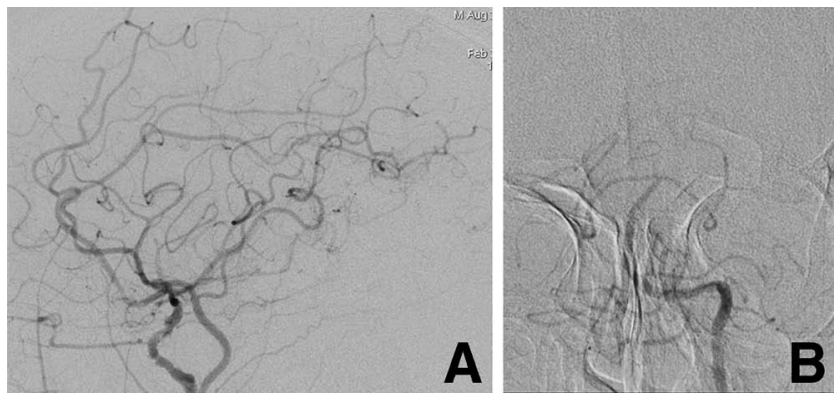

Fig. 4 A: Left carotid angiogram (lateral view) showing no posterior communicating arteries. B: Left subclavian artery angiogram (anteroposterior view) revealing that the basilar artery is perfused from the left vertebral artery only, which was reconstituted via the muscular and thyrocervical collateral vessels in the late phase.

4B). Detailed hemodynamics of the cerebral circulation was not evaluated because of his unstable neurological and general condition. We performed revascularization to prevent progressive ischemia.

Transarterial approaches via the left femoral and left brachial arteries were performed using 8-F and 4-F sheaths, respectively. An 8-F guide catheter (GuiderSoftip; Boston Scientific, Natick, Massachusetts, USA) was held with a 4-mm diameter snare wire (Amplatz GooseNeck Snare; Micro Therapeutics, Inc., Irvine, California, USA), which was approached from the left brachial artery at the left subclavian artery (SCA) (Fig. 5A). The non-visualized stump of the left VA ostium was extrapolated from the findings of partial calcification of the left 

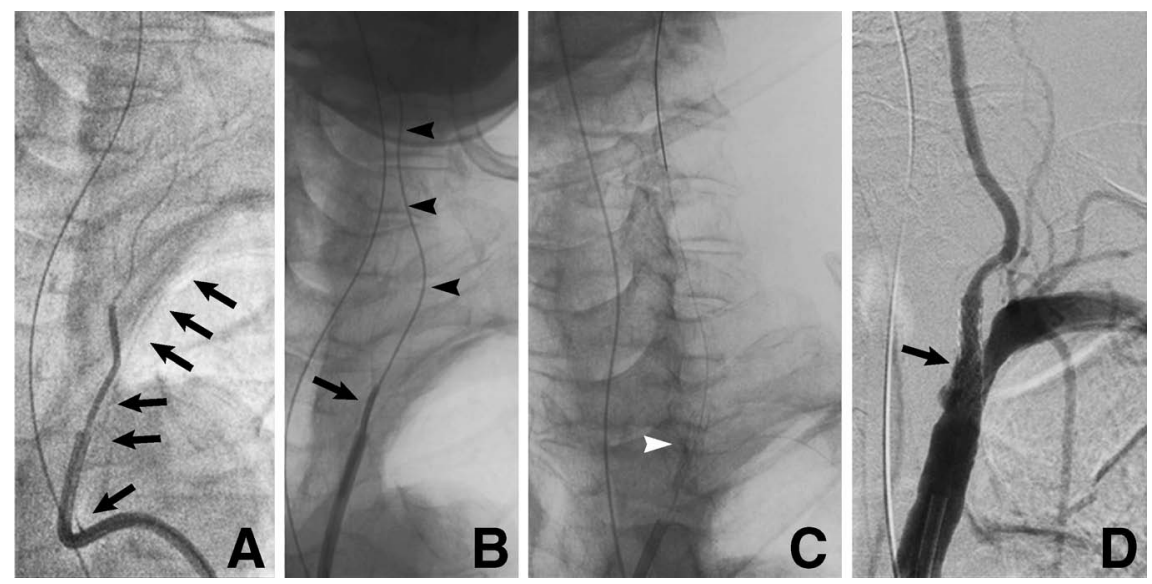

Fig. 5 Intraoperative angiograms of the left subclavian artery. A snare wire via the left brachial artery holds the guide catheter (A, arrows). A 0.035-inch guidewire is navigated across the occlusion to the distal patent vertebral artery at the level of the collateral vessels (B, arrowheads). A 4-F catheter is advanced over the guidewire across the occluded segment (B, arrow). After the exchanged microwire is used to remove the catheter, stent placement is performed (C, arrowhead). The occluded vertebral artery is restored, and anterograde flow is confirmed (D, arrow).

SCA on CT angiography. A 0.035-inch guidewire (Quick Flex; Kaneka Medix, Osaka) was passed through the occluded VA ostium and exchanged for a 0.014-inch guidewire (ASAHI CHIKAI; Asahi Intecc Co., Ltd., Nagoya, Aichi) with a 4-F catheter (Tempo; Cordis Corp., Bridgewater, New Jersey, USA) (Fig. 5B). Based on the normal vessel diameter, the occlusion was pre-dilated with a $3-\mathrm{mm}$ balloon (Gateway; Boston Scientific). A balloon expandable bare metal stent (4 $\mathrm{mm}$ diameter, $18 \mathrm{~mm}$ length; PALMAZ Genesis; Cordis Corp.) was then placed (Fig. 5C). Post-procedural angiography showed anterograde flow of the vertebrobasilar system (Fig. 5D).

The postoperative course of the patient was good with no recurrent ischemia, and his altered consciousness was resolved. He was transferred to another hospital for further management of the residual right hemiparesis at 14 days after the operation. Cerebral angiography obtained 3 months later revealed no significant restenosis of the lesion.

\section{Discussion}

The efficacy of percutaneous endovascular angioplasty for extracranial VA stenosis was recently reported in a systematic review. ${ }^{16)}$ Stenting of a longsegment extracranial VA occlusion was also reported.5) However, to our knowledge, no reports have described endovascular angioplasty of VA origin occlusion without visualization of the stump of the artery ostium. The present case report demonstrates that endovascular treatment of extracranial VA occlusion is a feasible treatment strategy even without visualization of the stump of the artery ostium. The major concerns with the use of endovascular angioplasty for VA occlusion are the inability to traverse a lesion, rupture or dissection of the VA, and stroke due to distal embolization. ${ }^{5)}$ To perform this procedure accurately, the following important factors should be considered.

The first factor is identification of the nonvisualized VA ostium. The origin of the VA is typically obscured by various embryological and acquired changes including atherosclerosis. ${ }^{4,10,11)}$ Misdirected wire manipulation may cause rupture or dissection of the artery. CT angiography evidence of partial calcification of the SCA and distal VA at the level of the collateral vessels provided useful landmarks for the VA ostium. We considered that the occlusion was caused by acute exacerbation of chronic VA ostium stenosis, as angiography revealed a retrograde patent VA via the collateral vessels. Therefore, pathologically, the partial calcification of the SCA was suspected to be part of the atherosclerotic change. Fortunately, angiography revealed only a few calcifications of the SCA. If no landmark of the VA origin can be identified, and the distance between the occlusion and the patent artery at the level of the collaterals is extremely long, a surgical procedure may be required rather than endovascular angioplasty. Reimplantation (transposition) of the VA on the common carotid artery, vertebral-to-carotid artery bypass, and vertebral endarterectomy are surgical options. ${ }^{2}{ }^{2}$ However, these surgical procedures pose a higher risk in patients in severe general condition, such as the congestive heart failure in our case.

The second factor is support of the guide catheter during the procedure. The guide catheter may be unstable if the distance between the aortic arch and the VA ostium is too short. Furthermore, the guide catheter can slip down due to resistance to wire manipulation that occurs as the wire passes the long segment occlusion. Previous reports have described 
a variety of techniques used to obtain stability of the guide catheter. $6,8,12,15,18,19)$ The buddy wire technique is a well-known and rapid technique, ${ }^{8)}$ although this can provide unsatisfactory support for the guide catheter during passage beyond the occlusion. We selected the pull-up technique with a GooseNeck Snare as it provides stable support for the guide catheter and a smooth approach to the lesion. ${ }^{18)}$ An ipsilateral retrograde brachial arterial approach with a long sheath may also be acceptable if the origin is at an ideal angle to the SCA. ${ }^{12)}$ However, in our case, the technical success of this technique was uncertain because the angle of VA ostium was not known.

Percutaneous revascularization with the aid of a distal embolic protection device for VA stenosis was previously reported. ${ }^{3,9,14,21)}$ However, a systematic review of percutaneous revascularization of extracranial VA stenosis found no differences in periprocedural stroke rates with or without protection devices. ${ }^{16)}$ Similarly, the endovascular treatment without an embolic protection device performed in our case did not result in procedural thromboembolic complication.

In the present case, the stent was placed to fully cover the VA ostium as the occlusion was at the level of the non-visualized VA ostium. This positioning of the stent with proximal protrusion into the large vessel can result in stent fracture. ${ }^{16,20)}$ However, stent fracture was reported to be associated with stent type, not vessel diameter, with the open-cell type of coronary stent exhibiting a high risk of fracture. ${ }^{17)}$ We used the present stent due to its closed-cell design, and its conformability and flexibility for tortuous vessels such as the renal artery, which was originally approved for the angioplasty.

In conclusion, we report a successful endovascular angioplasty of an extracranial VA occlusion without visualization of the stump of the artery ostium. CT angiography identified partial calcifications and a distal patent artery, so may be useful for estimated identification of the arterial ostium. The pullup technique with a GooseNeck Snare provides the advantages of good support and positioning of the guide catheter during the procedure.

\section{Conflicts of Interest Disclosure}

The authors have no personal financial or institutional interest in any of the drugs, materials, or devices in the article. All authors who are members of The Japan Neurosurgical Society (JNS) have registered online Self-reported COI Disclosure Statement Forms through the website for JNS members.

\section{References}

1) Caplan LR, Amarenco P, Rosengart A, Lafranchise EF, Teal PA, Belkin M, DeWitt LD, Pessin MS: Embolism from vertebral artery origin occlusive disease. Neurology 42: 1505-1512, 1992

2) Coert BA, Chang SD, Marks MP, Steinberg GK: Revascularization of the posterior circulation. Skull Base 15: 43-62, 2005

3) Eberhardt O, Naegele T, Raygrotzki S, Weller M, Ernemann U: Stenting of vertebrobasilar arteries in symptomatic atherosclerotic disease and acute occlusion: case series and review of the literature. J Vasc Surg 43: 1145-1154, 2006

4) Farrés MT, Magometschnigg H, Grabenwöger F, Trattnig S, Dock W, Heimberger K, Mühlbauer M, Lammer J: Stenoses of the first segment of the vertebral artery: difficulties in angiographic diagnosis. Neuroradiology 38: 6-10, 1996

5) Gupta R, Sivapatham T, Moskowitz SI, Srivastava S: Stenting of a symptomatic long-segment extracranial vertebral artery occlusion. J Neurointerv Surg 3: 54-56, 2011

6) Harada K, Nakahara I, Tanaka M, Iwamuro $Y$, Watanabe Y, Fujimoto M: [Therapeutic strategy and outcome of stenting for subclavian and innominate artery occlusive disease]. No Shinkei Geka 32: 151-158, 2004 (Japanese)

7) Hopkins LN, Budny JL: Complications of intracranial bypass for vertebrobasilar insufficiency. J Neurosurg 70: 207-211, 1989

8) Kizilkilic O: Vertebral artery origin stenting with buddy wire technique in tortuous subclavian artery. Eur J Radiol 61: 120-123, 2006

9) Ko YG, Park S, Kim JY, Min PK, Choi EY, Jung JH, Joung B, Choi D, Jang Y, Shim WH: Percutaneous interventional treatment of extracranial vertebral artery stenosis with coronary stents. Yonsei Med J 45: 629-634, 2004

10) Komiyama M, Morikawa T, Nakajima H, Nishikawa M, Yasui T: High incidence of arterial dissection associated with left vertebral artery of aortic origin. Neurol Med Chir (Tokyo) 41: 8-11, 2001

11) Kowada M, Yamaguchi K, Takahashi H: Fenestration of the vertebral artery with a review of 23 cases in Japan. Radiology 103: 343-346, 1972

12) Kusaka N, Tamiya T, Nishiguchi $M$, Takayama $K$, Nishiura T: Bilateral brachial pull-through technique for stenting in a patient with stenosis of the vertebral artery origin: technical case report. Neuroradiology 49: 837-839, 2007

13) Moufarrij NA, Little JR, Furlan AJ, Williams G, Marzewski DJ: Vertebral artery stenosis: long-term follow-up. Stroke 15: 260-263, 1984

14) Qureshi AI, Kirmani JF, Harris-Lane P, Divani AA, Ahmed S, Ebrihimi A, Al Kawi A, Janjua N: Vertebral artery origin stent placement with distal protection: technical and clinical results. AJNR Am J Neuroradiol 27: 1140-1145, 2006 
15) Ryer EJ, Oderich GS: Two-wire (0.014 \& 0.018-inch) technique to facilitate innominate artery stenting under embolic protection. J Endovasc Ther 17: 652-656, 2010

16) Stayman AN, Nogueira RG, Gupta R: A systematic review of stenting and angioplasty of symptomatic extracranial vertebral artery stenosis. Stroke 42: 2212-2216, 2011

17) Tsutsumi M, Kazekawa K, Onizuka M, Kodama T, Matsubara S, Aikawa H, Iko M, Nii K, Etou H, Tanaka A: Stent fracture in revascularization for symptomatic ostial vertebral artery stenosis. Neuroradiology 49: 253-257, 2007

18) Uda $T$, Murata K, Ichinose $T$, Kusakabe T, Sakaguchi $\mathrm{M}$ : [Usefulness of the goose neck snare for holding the guiding catheter for carotid artery stenting]. No Shinkei Geka 35: 673-676, 2007 (Japanese)

19) Uysal E, Caliskan C, Caymaz I, Orken DN, Basak M: Stenting of vertebral artery origin with the buddy wire technique in tortuous subclavian artery. A case report. Interv Neuroradiol 16: 175-178, 2010

20) Vajda Z, Miloslavski E, Güthe T, Fischer S, Albes G,
Heuschmid A, Henkes H: Treatment of stenoses of vertebral artery origin using short drug-eluting coronary stents: improved follow-up results. AJNR Am J Neuroradiol 30: 1653-1656, 2009

21) Werner $M$, Bräunlich $S$, Ulrich $M$, Bausback $Y$, Schuster J, Lukhaup A, Botsios S, Scheinert D, Schmidt A: Drug-eluting stents for the treatment of vertebral artery origin stenosis. J Endovasc Ther 17: 232-240, 2010

22) Wityk RJ, Chang HM, Rosengart A, Han WC, DeWitt LD, Pessin MS, Caplan LR: Proximal extracranial vertebral artery disease in the New England Medical Center Posterior Circulation Registry. Arch Neurol 55: 470-478, 1998

Address reprint requests to: Kouhei Nii, MD, Department of Neurosurgery, Fukuoka Kieikai Hospital, 5-11-5 Chihaya, Higashi-ku, Fukuoka 813-0044, Japan. e-mail:k.nii@cis.fukuoka-u.ac.jp 